\title{
Mott-insulator-like Bose-Einstein condensation in a tight-binding system of interacting bosons with a flat band
}

\author{
Hosho Katsura $\odot,{ }^{1,2,3}$ Naoki Kawashima, ${ }^{4}$ Satoshi Morita $\odot,{ }^{4}$ Akinori Tanaka $\odot,{ }^{5}$ and Hal Tasaki ${ }^{6, *}$ \\ ${ }^{1}$ Department of Physics, Graduate School of Science, The University of Tokyo, 7-3-1 Hongo, Tokyo 113-0033, Japan \\ ${ }^{2}$ Institute for Physics of Intelligence, The University of Tokyo, 7-3-1 Hongo, Tokyo 113-0033, Japan \\ ${ }^{3}$ Trans-scale Quantum Science Institute, The University of Tokyo, 7-3-1, Hongo, Tokyo 113-0033, Japan \\ ${ }^{4}$ The Institute for Solid State Physics, The University of Tokyo, Kashiwa, Chiba 277-8581, Japan \\ ${ }^{5}$ Department of General Education, National Institute of Technology, Ariake College, Omuta, Fukuoka 836-8585, Japan \\ ${ }^{6}$ Department of Physics, Gakushuin University, Mejiro, Toshima-ku, Tokyo 171-8588, Japan
}

(Received 14 May 2021; revised 13 August 2021; accepted 13 August 2021; published 25 August 2021)

\begin{abstract}
We propose a class of tight-binding systems of interacting bosons with a flat band, which are exactly solvable in the sense that one can explicitly write down the unique ground state. The ground state is expressed in terms of local creation operators and apparently resembles that of a Mott insulator. Based on an exact representation in terms of a classical loop-gas model, we conjecture that the ground state may exhibit quasi Bose-Einstein condensation (BEC) and genuine BEC in dimension 2 and in dimension 3 or higher, respectively, still keeping Mott-insulator-like character. Our Monte Carlo simulation of the loop-gas model strongly supports this conjecture, i.e., the ground state undergoes a Kosterlitz-Thouless transition and exhibits quasi-BEC in two dimensions.
\end{abstract}

DOI: 10.1103/PhysRevResearch.3.033190

\section{INTRODUCTION}

Tight-binding models of interacting particles with a flat band, i.e., a set of highly degenerate single-particle energy eigenstates, have been studied intensively over the decades. Flat-band systems not only serve as idealized models of materials with a narrow band but also provide a theoretical playground for investigating various collective phenomena arising from the interplay between particle motion and interactions. This is because the effect of interactions is magnified due to the flatness of the band. Such an approach was fruitful in the study of the origin of ferrimagnetism [1] and ferromagnetism [2-5] in the Hubbard model. See Ref. [6] for a review. For the formation of a Wigner crystal and the effect of the change in the density in bosonic systems with a flat band, see Refs. [7-11]. The recent proposal that the nearly flat band in twisted bilayer graphene supports superconductivity is intriguing [12].

In this paper we propose a class of tight-binding systems of interacting bosons with a flat lowest band. The model is based on the construction in Ref. [13] of flat-band Hubbard models and can be regarded as a spinless version of the models studied in Ref. [14]. We write down the ground state of the model explicitly as in (5) and prove that it is the unique ground state. The expression suggests that the ground state is essentially

\footnotetext{
*Corresponding author: hal.tasaki@gakushuin.ac.jp

Published by the American Physical Society under the terms of the Creative Commons Attribution 4.0 International license. Further distribution of this work must maintain attribution to the author(s) and the published article's title, journal citation, and DOI.
}

different from that of a noninteracting system and resembles that of a Mott insulator.

In spite of the simple expression, the property of the ground state is nontrivial and rich. By examining representations of the norm and the correlation functions in terms of a classical loop-gas model, we conjecture that the ground states may exhibit quasi off-diagonal long-range order (ODLRO) in two dimensions and genuine ODLRO in three or higher dimensions. We present some results of Monte Carlo simulation of the loop-gas model, which strongly indicates that the two-dimensional model undergoes a Kosterlitz-Thouless (KT) transition and exhibits quasi-ODLRO in its ground states.

We note that a class of states (without parent Hamiltonians) very similar to ours was proposed and examined in Ref. [15]. It was found that these states do not exhibit off-diagonal (quasi) long-range order.

It was found in a two-component system of bosons that one component may exhibit Bose-Einstein condensation (BEC) while the other is in the Mott insulating state [16]. This is different from our Mott-insulator-like ground state (5), which consists only of the states created by the $\hat{b}_{u}^{\dagger}$ operators. Note also that our ground state in the BEC phase, although solidlike, is not a supersolid since there is no spontaneous breakdown of translation symmetry [17-19]. It is a challenging problem to design a similar exactly solvable model that has supersolid ground states.

We stress that our ground states maintain a Mott-insulatorlike nature even when they exhibit (quasi-)ODLRO. This is most clearly seen in the anomalously small particlenumber fluctuation observed in a specific setting. See Sec. VI (in particular, Fig. 8). This does not mean, however, that the ground states describe genuine Mott insulators. See Sec. V. 
(a)

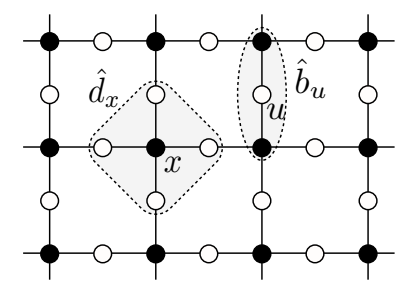

(b)

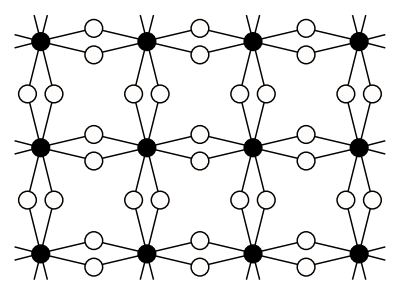

FIG. 1. (a) The lattice $\Lambda$ in the case $(\mathcal{E}, \mathcal{B})$ is the square lattice $(d=2, p=1)$. The black and white circles denote the sites in $\mathcal{E}$ and $\mathcal{I}$, respectively. The states corresponding to the $\hat{b}$ and $\hat{d}$ operators are also shown. (b) The lattice $\Lambda$ obtained from the square lattice in which neighboring sites are connected by two bonds $(d=2, p=2)$.

It would be exciting if our exactly solvable model provides an example of a novel exotic phase of matter where a Mott-insulator-like nature and (quasi-)ODLRO coexist. Although we are not able to give a definite conclusion at the moment, we find it rather likely that (unfortunately) our Mottinsulator-like condensate is smoothly connected to ordinary Bose-Einstein condensate realized, e.g., in noninteracting systems. See Sec. VI.

We nevertheless believe that it is of essential importance that this class of exactly solvable models of strongly interacting bosons has been discovered. We hope that this paper opens a new direction in the research of quantum many-body systems.

\section{THE MODEL AND THE EXACT GROUND STATE}

Let $(\mathcal{E}, \mathcal{B})$ be a finite lattice, where $\mathcal{E}$ is the set of sites and $\mathcal{B}$ is the set of bonds. A bond is an unoriented segment that connects two distinct sites in $\mathcal{E}$. We allow multiple bonds to connect the same pair of sites. At the center of each bond $b \in \mathcal{B}$, we take a new site and denote it as $u_{b}$. We let $\mathcal{I}$ be the set of all sites $u_{b}$ with $b \in \mathcal{B}$ and consider the decorated lattice $\Lambda=\mathcal{E} \cup \mathcal{I}$. We typically choose $\mathcal{E}$ to be the set of sites of the $d$-dimensional hypercubic lattice with periodic boundary conditions and take $p$ distinct bonds connecting a pair of neighboring sites. See Fig. 1 for the resulting decorated lattices.

We shall define a tight-binding model of bosons on $\Lambda$. We denote by $\hat{a}_{r}^{\dagger}$ and $\hat{a}_{r}$ the creation and annihilation operators, respectively, of a boson at site $r \in \Lambda$. They satisfy the canonical commutation relations $\left[\hat{a}_{r}, \hat{a}_{s}\right]=0$ and $\left[\hat{a}_{r}, \hat{a}_{s}^{\dagger}\right]=\delta_{r, s}$ for any $r, s \in \Lambda$. The number operator is defined as $\hat{n}_{r}=\hat{a}_{r}^{\dagger} \hat{a}_{r}$. We denote by $\left|\Phi_{\text {vac }}\right\rangle$ the state without any bosons, i.e., the unique normalized state such that $\hat{a}_{r}\left|\Phi_{\text {vac }}\right\rangle=0$ for any $r \in \Lambda$. For each $x \in \mathcal{E}$, we define

$$
\hat{d}_{x}:=\zeta \hat{a}_{x}+\sum_{u \in \mathcal{N}(x)} \hat{a}_{u},
$$

where $\zeta>0$ is a model parameter and $\mathcal{N}(x)$ is the set of sites in $\mathcal{I}$ that are on the bonds connected to $x$.

We consider the Hamiltonian $\hat{H}=\hat{H}_{\text {hop }}+\hat{H}_{\text {int }}$ with the hopping Hamiltonian

$$
\hat{H}_{\text {hop }}:=t \sum_{x \in \mathcal{E}} \hat{d}_{x}^{\dagger} \hat{d}_{x},
$$

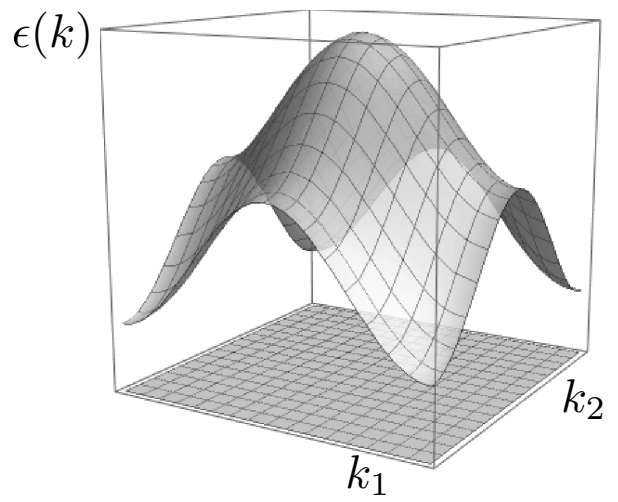

FIG. 2. The single-particle energy eigenvalue $\epsilon(k)$ as a function of the wave-number vector $k=\left(k_{1}, k_{2}\right)$ for the models defined on the square lattice $(\mathcal{E}, \mathcal{B})$ as in Fig. 1 . Here, $k_{j}(j=1,2)$ runs between $-\pi$ and $\pi$. There are flat bands (formed by the $\hat{b}$ states) with zero energy and a dispersive band (formed by the $\hat{d}$ states) with width $4 p t$. The band gap is equal to $\zeta^{2} t$. See Appendix A and, in particular, Eq. (A10).

where $t>0$, and the interaction Hamiltonian

$$
\hat{H}_{\text {int }}:=\frac{U}{2} \sum_{u \in \mathcal{I}} \hat{n}_{u}\left(\hat{n}_{u}-1\right),
$$

where $U>0$. Our model is characterized by the three parameters $\zeta, t$, and $U$. Note that $\hat{H}_{\text {hop }}$ is rewritten in the standard form as $\hat{H}_{\text {hop }}=\sum_{r, s \in \Lambda} t_{r, s} \hat{a}_{r}^{\dagger} \hat{a}_{s}$, where the amplitude $t_{r, s}$ describes hopping between the nearest and some of the next-nearest-neighbor sites, and on-site potentials. Note also that $\hat{H}_{\text {int }}$ describes on-site repulsive interaction only on sites in $\mathcal{I}$.

The hopping Hamiltonian $\hat{H}_{\text {hop }}$ describes a tight-binding model with a flat band. To see this, we define

$$
\hat{b}_{u}:=\frac{1}{\sqrt{2+\zeta^{2}}}\left(\zeta \hat{a}_{u}-\hat{a}_{x}-\hat{a}_{y}\right),
$$

for $u \in \mathcal{I}$, where $x, y \in \mathcal{E}$ are the sites connected by the bond corresponding to $u$. One can easily verify the orthogonality $\left[\hat{d}_{x}, \hat{b}_{u}^{\dagger}\right]=0$ for any $x \in \mathcal{E}$ and $u \in \mathcal{I}$. This means that $\hat{H}_{\text {hop }} \hat{b}_{u}^{\dagger}\left|\Phi_{\text {vac }}\right\rangle=0$ for any $u \in \mathcal{I}$. Since $\hat{H}_{\text {hop }} \geqslant 0$ and the states $\hat{b}_{u}^{\dagger}\left|\Phi_{\text {vac }}\right\rangle$ with $u \in \mathcal{I}$ are linearly independent, we see that $\hat{H}_{\text {hop }}$ has $|\mathcal{I}|$ independent single-particle ground states (with zero energy). See Fig. 2 and Appendix A for more about the single-particle energy eigenvalues.

Let us state our first theorem, which characterizes the ground state of the model.

Theorem 1. Consider the above model with particle number $N=|\mathcal{I}|$. For any $\zeta>0, t>0$, and $U>0$, the ground state of $\hat{H}$ is unique, has vanishing energy, and is written as

$$
\left|\Phi_{\mathrm{GS}}\right\rangle=\left(\prod_{u \in \mathcal{I}} \hat{b}_{u}^{\dagger}\right)\left|\Phi_{\mathrm{vac}}\right\rangle .
$$

Note that, in the ground state (5), exactly one particle is associated with the adjacent three sites including $u \in \mathcal{I}$. This suggests that the particles are distributed almost uniformly over the lattice, and the state resembles the ground state of a "solid" or, more precisely, a Mott insulator. However, the property of the ground state turns out to be much richer than a 
simple Mott insulator. In fact, we shall argue in Sec. V that the ground state does not correspond to a genuine Mott insulator when it exhibits (quasi-)ODLRO.

The proof of Theorem 1 is an easy application of the technique developed in Refs. $[4,5,13]$ for the (fermionic) Hubbard model.

Proof of Theorem 1. It follows from the definitions that $\hat{H}\left|\Phi_{\mathrm{GS}}\right\rangle=0$. Since $\hat{H} \geqslant 0$, this proves that $\left|\Phi_{\mathrm{GS}}\right\rangle$ is a ground state. We only need to prove that it is the unique ground state.

We first note that any single-particle state can be expressed by a linear combination of the operators $\hat{b}_{u}^{\dagger}$ with $u \in \mathcal{I}$ and $\hat{d}_{x}^{\dagger}$ with $x \in \mathcal{E}$. This follows by observing that $\hat{b}_{u}^{\dagger}$ and $\hat{d}_{x}^{\dagger}$ are all linearly independent, and there are exactly $|\mathcal{I}|+|\mathcal{E}|=|\Lambda|$ operators. We thus see that any $N$-particle state is written as a linear combination of the basis states $\left|\Phi_{\boldsymbol{n}}\right\rangle=\left\{\prod_{u \in \mathcal{I}}\left(\hat{b}_{u}^{\dagger}\right)^{n_{u}}\right\}\left\{\prod_{x \in \mathcal{E}}\left(\hat{d}_{x}^{\dagger}\right)^{n_{x}}\right\}\left|\Phi_{\text {vac }}\right\rangle$, where the "occupation number" $\boldsymbol{n}=\left(n_{r}\right)_{r \in \Lambda}$ satisfies $\sum_{r \in \Lambda} n_{r}=N$.

Our proof is based on the standard argument for frustration-free Hamiltonians. Let $|\Psi\rangle$ be an arbitrary state with $N=|\mathcal{I}|$ particles such that $\hat{H}|\Psi\rangle=0$, and expand it as $|\Psi\rangle=\sum_{n} \alpha_{n}\left|\Phi_{n}\right\rangle$. Noting that $\hat{d}_{x}^{\dagger} \hat{d}_{x} \geqslant 0$ and $\hat{n}_{u}\left(\hat{n}_{u}-1\right)=$ $\left(\hat{a}_{u}^{\dagger}\right)^{2}\left(\hat{a}_{u}\right)^{2} \geqslant 0$, we see that $\hat{d}_{x}^{\dagger} \hat{d}_{x}|\Psi\rangle=0$ and $\left(\hat{a}_{u}^{\dagger}\right)^{2}\left(\hat{a}_{u}\right)^{2}|\Psi\rangle=$ 0 for all $x \in \mathcal{E}$ and $u \in \mathcal{I}$. These relations further imply that $\hat{d}_{x}|\Psi\rangle=0$ for all $x \in \mathcal{E}$ and $\left(\hat{a}_{u}\right)^{2}|\Psi\rangle=0$ for all $u \in \mathcal{I}$. The first condition implies that $\alpha_{n}=0$ whenever $n_{x} \neq 0$ for some $x \in \mathcal{E}$. Thus the ground state contains only the $\hat{b}$ states. Then the second condition implies that $\alpha_{n}=0$ whenever $n_{u} \geqslant 2$ for some $u \in \mathcal{I}$. This means that $|\Psi\rangle$ is a constant multiple of $\left|\Phi_{\mathrm{GS}}\right\rangle$.

\section{GROUND-STATE PHASE TRANSITION}

We first derive an exact loop-gas model representation of the ground state. Further details of the derivation are given in Appendix B. Note that in this quantum-classical correspondence, unlike in the standard correspondence via path integral, a $d$-dimensional quantum state is mapped to a $d$ dimensional classical system. This is a peculiar point about our model and is analogous to the loop-gas representations of the Affleck-Kennedy-Lieb-Tasaki (AKLT) model [20] and the Kitaev model [21].

Note first that the $\hat{b}$ operators satisfy the commutation relations

$$
\left[\hat{b}_{u}, \hat{b}_{v}^{\dagger}\right]= \begin{cases}1 & u=v \\ 2 \beta & u \approx v \\ \beta & u \sim v \\ 0 & \text { otherwise }\end{cases}
$$

where

$$
\beta:=\left(2+\zeta^{2}\right)^{-1} .
$$

Here, $u \approx v$ indicates that the bonds corresponding to $u$ and $v$ connect the same pair of sites, and $u \sim v$ indicates that the bonds for $u$ and $v$ share a single common site. By repeatedly using (6), one finds that the normalization factor $\left\langle\Phi_{\mathrm{GS}} \mid \Phi_{\mathrm{GS}}\right\rangle$ is represented as [15]

$$
\left\langle\Phi_{\mathrm{GS}} \mid \Phi_{\mathrm{GS}}\right\rangle=\sum_{\mathcal{L}} \beta^{|\mathcal{L}|},
$$

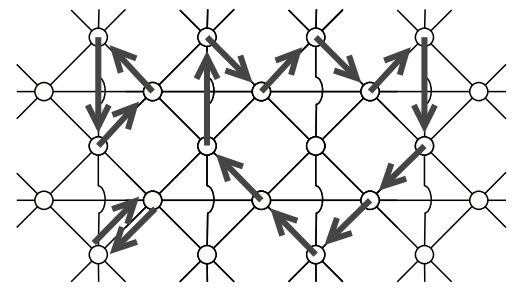

FIG. 3. A typical configuration of loops on $\mathcal{I}$ corresponding to the lattice in Fig. 1(a). By an arrow from site $u$ to $v$ we indicate the commutator $\left[\hat{b}_{u}, \hat{b}_{v}^{\dagger}\right]$.

where the sum is over all possible sets $\mathcal{L}=\left\{\ell_{1}, \ldots, \ell_{n}\right\}$ with $n=0,1,2, \ldots$ of oriented loops. See Fig. 3. By an oriented loop of length $m$, we mean a sequence $\ell=\left(u_{1}, \ldots, u_{m}\right)$ of $m$ distinct sites in $\mathcal{I}$ such that $u_{j} \approx u_{j+1}$ or $u_{j} \sim u_{j+1}$ for $j=$ $1, \ldots, m$, where we set $u_{m+1}=u_{1}$. Here, we identify the new sequence obtained by the shift $u_{j} \rightarrow u_{j+1}$ for $j=1, \ldots, m$ with the original sequence. This means that, for $u_{1}, u_{2} \in \mathcal{I}$ such that $u_{1} \sim u_{2}$, there is a unique loop, $\ell=\left(u_{1}, u_{2}\right)$, of length 2 , while for $u_{1}, u_{2}, u_{3} \in \mathcal{I}$ such that $u_{1} \sim u_{2}, u_{2} \sim$ $u_{3}$, and $u_{3} \sim u_{1}$, there are two loops, $\ell=\left(u_{1}, u_{2}, u_{3}\right)$ and $\bar{\ell}=\left(u_{3}, u_{2}, u_{1}\right)$, of length 3 . We also assume that, in any set $\mathcal{L}=\left\{\ell_{1}, \ldots, \ell_{n}\right\}$, no loops share a common site. To take into account the factor 2 in (6), we interpret $u, v \in \mathcal{I}$ with $u \approx v$ as being connected via two distinct paths and properly overcount loops according to this interpretation. Finally, we wrote $|\mathcal{L}|=\sum_{j=1}^{n}\left|\ell_{j}\right|$, where $|\ell|$ denotes the length of a loop $\ell$. See Appendix B for details.

We can derive a similar representation for correlation functions. Noting that $\hat{a}_{u}\left|\Phi_{\mathrm{GS}}\right\rangle=\zeta \sqrt{\beta}\left(\prod_{w \neq u} \hat{b}_{w}^{\dagger}\right)\left|\Phi_{\text {vac }}\right\rangle$ for $u \in$ $\mathcal{I}$, we find that

$$
\left\langle\Phi_{\mathrm{GS}}\left|\hat{a}_{v}^{\dagger} \hat{a}_{u}\right| \Phi_{\mathrm{GS}}\right\rangle=\zeta^{2} \beta \sum_{\mathcal{L}, \omega: u \rightarrow v}^{\prime} \beta^{|\mathcal{L}|+|\omega|},
$$

where $\mathcal{L}$ is again summed over sets of oriented loops and $\omega$ is summed over all self-avoiding walks connecting $u$ to $v$, i.e., a sequence $\omega=\left(u_{0}, \ldots, u_{m}\right)$ of $m+1$ distinct sites in $\mathcal{I}$ such that $u_{0}=u, u_{m}=v$, and $u_{j} \approx u_{j+1}$ or $u_{j} \sim u_{j+1}$ for $j=0, \ldots, m-1$. The length of the walk is defined as $|\omega|=m$. The prime in the sum indicates that $\omega$ and loops in $\mathcal{L}$ do not share common sites. By combining (8) and (9), we see that the off-diagonal correlation is represented as

$$
\left\langle\hat{a}_{v}^{\dagger} \hat{a}_{u}\right\rangle_{\mathrm{GS}}=\frac{\left\langle\Phi_{\mathrm{GS}}\left|\hat{a}_{v}^{\dagger} \hat{a}_{u}\right| \Phi_{\mathrm{GS}}\right\rangle}{\left\langle\Phi_{\mathrm{GS}} \mid \Phi_{\mathrm{GS}}\right\rangle}=\frac{\zeta^{2} \beta \sum_{\mathcal{L}, \omega: u \rightarrow v}^{\prime} \beta^{|\mathcal{L}|+|\omega|}}{\sum_{\mathcal{L}} \beta^{|\mathcal{L}|}} .
$$

The correlation function $\left\langle\hat{a}_{r}^{\dagger} \hat{a}_{s}\right\rangle_{\mathrm{GS}}$ with general $r, s \in \Lambda$ has a similar (but slightly more complicated) representation and should behave almost similarly as $\left\langle\hat{a}_{v}^{\dagger} \hat{a}_{u}\right\rangle_{\mathrm{GS}}$, especially when the distance between $r$ and $s$ is large.

The above summations over loops and walks are in general nontrivial and hardly evaluated explicitly. When the basic lattice $(\mathcal{E}, \mathcal{B})$ is a chain with bonds connecting nearest-neighbor sites, i.e., $d=p=1$ in the notation of Sec. II, one can evaluate the summations by using, e.g., the transfer-matrix method 
to show for a long enough chain that

$$
\left\langle\hat{a}_{v}^{\dagger} \hat{a}_{u}\right\rangle_{\mathrm{GS}}=C\left(\frac{2 \beta}{1+\sqrt{1+4 \beta^{2}}}\right)^{|u-v|} \leqslant C 2^{-|u-v|},
$$

where $C=\zeta^{2} \beta / \sqrt{1+4 \beta^{2}}$. The correlation always decays exponentially, and the ground state is disordered.

Let us turn to the models in higher dimensions. When $\beta$ is small (or $\zeta$ is large), we can easily prove that the corresponding loop-gas model is in the disordered phase, where configurations with only small loops are dominant. This corresponds to a disordered ground state that describes a Mott insulator. To see this, we relax the constraint $\mathcal{L} \cap \omega=\emptyset$ in (9) to get

$$
\begin{aligned}
\left\langle\Phi_{\mathrm{GS}}\left|\hat{a}_{v}^{\dagger} \hat{a}_{u}\right| \Phi_{\mathrm{GS}}\right\rangle & \leqslant \zeta^{2} \beta \sum_{\mathcal{L}, \omega: u \rightarrow v} \beta^{|\mathcal{L}|+|\omega|} \\
& =\zeta^{2} \beta\left\langle\Phi_{\mathrm{GS}} \mid \Phi_{\mathrm{GS}}\right\rangle \sum_{\omega: u \rightarrow v} \beta^{|\omega|}
\end{aligned}
$$

This implies

$$
\left\langle\hat{a}_{v}^{\dagger} \hat{a}_{u}\right\rangle_{\mathrm{GS}} \leqslant \zeta^{2} \beta \sum_{\omega: u \rightarrow v} \beta^{|\omega|}=\zeta^{2} \beta \sum_{n=\operatorname{dist}(u, v)}^{\infty} \Omega_{u, v}(n) \beta^{n},
$$

where $\Omega_{u, v}(n)$ is the total number of self-avoiding walks of length $n$ that connect $u$ and $v$ and $\operatorname{dist}(u, v)$ is the minimum length of such walks. For $u \in \mathcal{I}$, let $s_{u}$ and $d_{u}$ be the numbers of $v$ such that $u \sim v$ and $u \approx v$, respectively. We let $v$ be a constant such that $s_{u}+2 d_{u} \leqslant v+1$ for any $u \in \mathcal{I}$. Then we have $\Omega_{u, v}(n) \leqslant(v+1) v^{n-2}$. [We give the following proof: There are at most $(v+1)$ choices for the first step and at most $v$ choices for each of the following $n-2$ steps. There is no choice in the final step because the walk ends at $v$.] This bound, with (13), implies that the correlation decays exponentially for sufficiently small $\beta$, or, equivalently, sufficiently large $\zeta$.

Theorem 2. Let $\zeta$ be such that $\nu \beta=v /\left(2+\zeta^{2}\right)<1$. Then we have for any $u, v \in \mathcal{I}$ that

$$
\left\langle\hat{a}_{v}^{\dagger} \hat{a}_{u}\right\rangle_{\mathrm{GS}} \leqslant \frac{\zeta^{2} \beta(v+1)}{v^{2}(1-v \beta)}(\nu \beta)^{\operatorname{dist}(u, v)} .
$$

When the dimension is larger than 1 and $\beta$ is sufficiently large, on the other hand, it is expected that the loop-gas model is in the percolating phase where a macroscopically large loop (or a walk) appears. The transition can be characterized by the divergence of the "static structure factor"

$$
S:=\left(\zeta^{2} \beta\right)^{-1} \sum_{v \in \mathcal{I}}\left\langle\hat{a}_{v}^{\dagger} \hat{a}_{u}\right\rangle_{\mathrm{GS}}
$$

If the critical value of $\beta$ for the transition is less than $1 / 2$, which is the upper bound for $\beta$, the ground state undergoes a phase transition.

Let us first examine the standard large- $d$ (or mean-field) approximation, in which one fixes $\tilde{\beta}=v \beta$ and lets $d \uparrow \infty$ (and hence $v \uparrow \infty$ ). It is well known that, for $\tilde{\beta}<1$, one can neglect contributions from loops in this limit. See Appendix
C. Then $S$ is evaluated for any $\tilde{\beta}<1$ as

$$
S \simeq \sum_{\omega: u \rightarrow .} \beta^{|\omega|}=\sum_{n=0}^{\infty} v^{n} \beta^{n}=\frac{1}{1-\tilde{\beta}},
$$

where $\omega$ is summed over all self-avoiding walks (with an arbitrary length) that start from $u$. We here noted that the number of walks is almost equal to $v^{n}$. The structure factor $S$ diverges as $\beta$ approaches the critical value $v^{-1}$.

The existence of a phase transition is also suggested by a more careful examination of the loop-gas model. Recall that, in (8) or (9), every loop of length 3 or more is summed exactly twice with different orientations. This is equivalent to considering unoriented loops, but with an extra factor 2 for each loop. We thus see that our loop-gas model resembles that obtained from the high-temperature expansion of an $\mathrm{O}(2)$ symmetric classical ferromagnetic spin system at a finite temperature. The $\mathrm{O}(2)$ symmetry corresponds to the $\mathrm{U}(1)$ phase symmetry in the original quantum system. From this analogy we conjecture that in dimensions 3 or higher the ground state $\left|\Phi_{\mathrm{GS}}\right\rangle$ may exhibit BEC (where $\left\langle\hat{a}_{v}^{\dagger} \hat{a}_{u}\right\rangle_{\mathrm{GS}}$ has long-range order) while in two dimensions it may exhibit a quasi-BEC (where $\left\langle\hat{a}_{v}^{\dagger} \hat{a}_{u}\right\rangle_{\mathrm{GS}}$ shows power-law decay), both at sufficiently large $\beta$.

To prove the existence of BEC is extremely difficult, if not impossible, since we need to show the breakdown of a continuous symmetry. See Ref. [22] and references therein for rare cases where the existence of BEC can be established rigorously by using the method of reflection positivity. (A readable account of the reflection positivity method can be found in Ref. [6].) In the present model, we have a rigorous result only for the system defined on a tree, where one can apply standard techniques (see, e.g., Refs. [23,24]). See Appendix D.

\section{NUMERICAL EVIDENCE OF A KOSTERLITZ-THOULESS TRANSITION}

To see whether the ground state exhibits a phase transition, we carried out a Monte Carlo simulation of the corresponding loop-gas model by using the worm algorithm [25]. We focus on the two-dimensional models constructed from the $L \times L$ square lattice with bond number $p=1$ and 2 and with periodic boundary conditions. See Fig. 1.

A central quantity that we measured is the static structure factor $S$ defined in (15). When the correlation $\left\langle\hat{a}_{v}^{\dagger} \hat{a}_{u}\right\rangle_{\mathrm{GS}}$ becomes long ranged, the structure factor should diverge as a function of $L$. Specifically, $S=O\left(L^{d}\right)$ if the system possesses ODLRO, and $S=O\left(L^{d-\eta}\right)$ if it has quasi-ODLRO characterized by the correlation-decay exponent $\eta$, while $S=O(1)$ in the disordered phase. In particular, $\eta=1 / 4$ at the KT transition point with the multiplicative logarithmic correction of $(\log L)^{1 / 8}[26]$.

To capture more specific features of the KT transition, we also measured the helicity modulus, which is proportional to the superfluid density. It can be computed as the Monte Carlo average of the squared total winding number of all loops $[15,27]$,

$$
\Upsilon:=\left\langle\left(\sum_{\ell \in \mathcal{L}} w_{\ell}\right)^{2}\right\rangle_{\mathrm{MC}}=\left\langle\sum_{\ell \in \mathcal{L}} w_{\ell}^{2}\right\rangle_{\mathrm{MC}},
$$




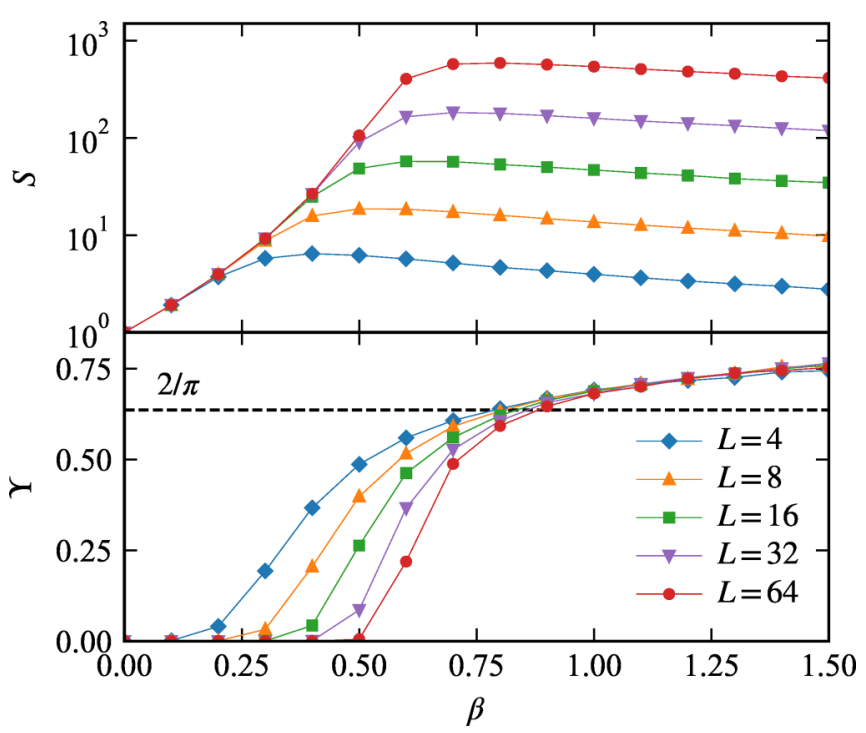

FIG. 4. The static structure factor $S$ (top) and the helicity modulus (bottom) as functions of $\beta$ for $p=1$. The horizontal line in the bottom panel indicates the universal jump $2 / \pi$, the thermodynamic value expected for the KT transition point.

where the summation is over all loops in the loop-gas configuration $\mathcal{L}$ and $w_{l}$ is the winding number of the loop $l$ around the horizontal direction of the lattice. As we pass the KT transition point entering the quasi-ODLRO phase, this quantity is expected to show a discontinuous jump from zero to the universal value $2 / \pi$, and keep increasing afterwards.

Let us start from the simplest model with $p=1$. The top panel of Fig. 4 shows the $\beta$ dependence of the static structure factor $S$ for varying system sizes up to $L=64$. Clearly, we do not observe any singularity in the region $\beta \leqslant 1 / 2$, where the classical-quantum correspondence is valid. The data, however, seem to indicate that the system, as a classical loop-gas model, has a phase transition beyond $\beta=1 / 2$.

The bottom panel of Fig. 4 shows the $\beta$ dependence of the helicity modulus $\Upsilon$. It can be seen from the figure that $\Upsilon$ goes from zero to some finite value greater than the expected jump, indicating that a KT transition takes place near $\beta \sim 1$. This is in contrast to the case studied in Ref. [15]. We shall recall, however, that the loop-gas model with $\beta \sim 1$ does not correspond to a quantum mechanical ground state.

Having observed that the model with $p=1$ does not exhibit a transition (in the range $\beta<1 / 2$ that is relevant for us), let us focus on the model with $p=2$. Figure 5 shows the $\beta$ dependence of the structure factor (top panel) and the helicity modulus (bottom panel) for varying system sizes up to $L=64$ in the loop-gas model corresponding to $p=2$. Clearly, the static structure factor $S$ shows a diverging behavior for large but not too large values of $\beta$. The helicity modulus $\Upsilon$ also shows an increase from zero to some finite value greater than the expected universal jump $\pi / 2$, strongly suggesting the presence of a KT transition in the physically meaningful region $\beta<1 / 2$.

Further evidence of a transition can be obtained from the size dependence of the structure factor, shown in Fig. 6. For small values of $\beta$, before the slope of the curve reaches $7 / 4$, it is not a straight line but bends and starts converging to a

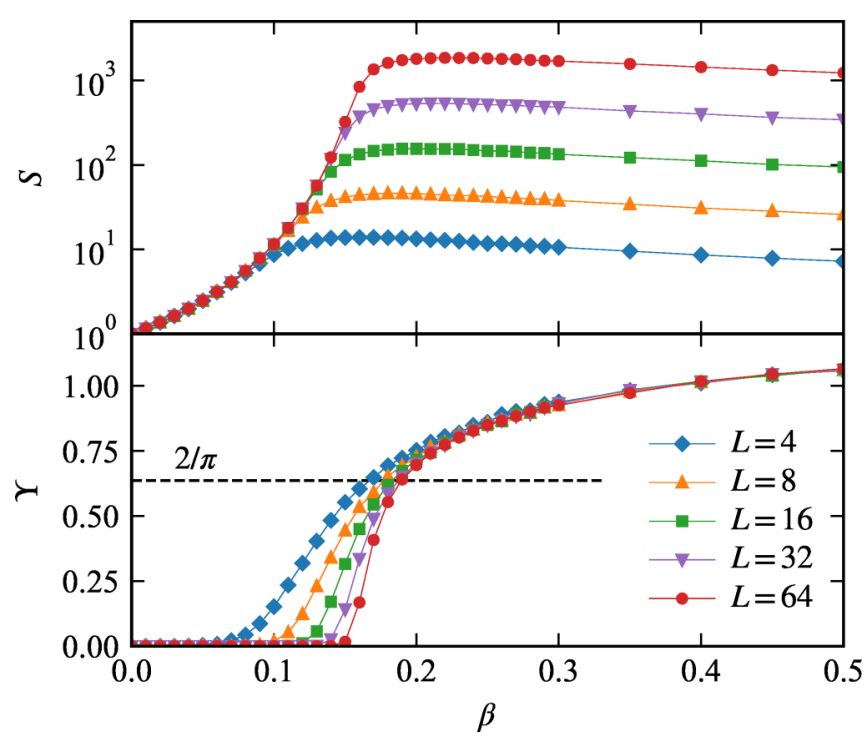

FIG. 5. The static structure factor $S$ (top) and the helicity modulus (bottom) as functions of $\beta$ for $p=2$. The horizontal line in the bottom panel indicates the universal jump $2 / \pi$, the thermodynamic value expected for the KT transition point.

finite value, as seen for $\beta=0.15$. As we increase $\beta$, the curve becomes a straight line with slope approximately equal to $7 / 4$ ( $\beta=0.20$ in the figure). Further increase in $\beta$ brings the slope of the straight line to a value greater than $7 / 4$ ( $\beta=0.5$ in the figure). To estimate the slope of the straight part of the curve more quantitatively, we analyzed the data with or without the expected logarithmic correction, i.e., fit the form $L^{2-\eta}$ or $(\log L)^{1 / 8} L^{2-\eta}$ to the data regarding the exponent $\eta$ as a fitting parameter, although according to Ref. [28] it is not clear whether including such a logarithmic correction would improve the estimates or not. The inset shows the result of the fitting with or without the logarithmic factor. From this result, we may conclude that the KT transition takes place at $\beta=0.20(1)$, which is consistent with the universal jump in the bottom panel of Fig. 5 considering the expected slow $\left[\sim(\log L)^{-1}\right]$ convergence of $\Upsilon$ at the transition point.

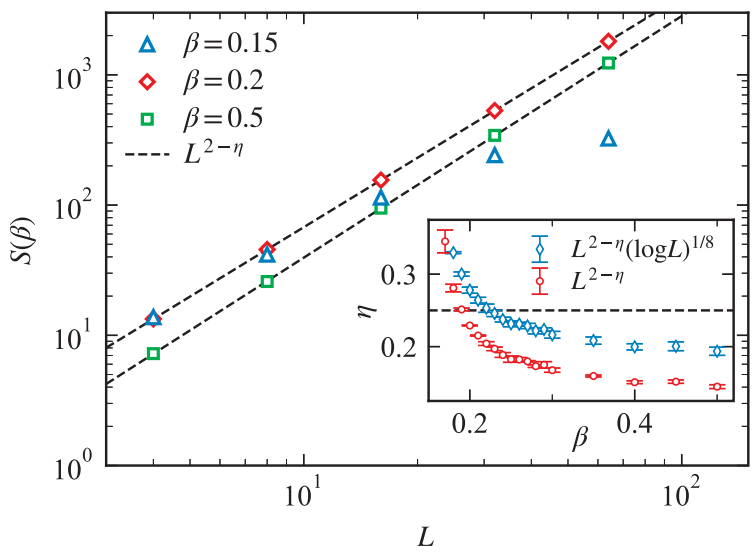

FIG. 6. The size dependence of the structure factor $S$. The dashed lines are the power function fitting. The inset shows the estimates of the critical exponent $\eta$ obtained by fitting with the simple power function (blue) and the power function with the expected logarithmic correction (red). 


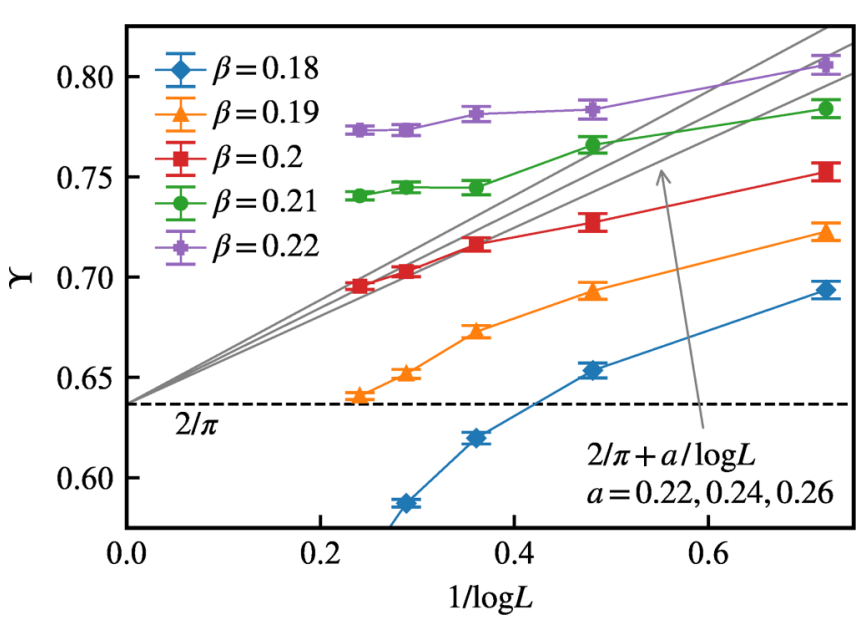

FIG. 7. The size dependence of the helicity modulus near the transition point for $p=2$. Straight lines are guides for the eye.

To get additional evidence of a KT transition, we examined this size dependence of the helicity modulus near the transition point in more detail. As shown in Fig. 7, we plot $\Upsilon$ as a function of $1 / \ln L$ around the critical value of $\beta$. At $\beta=0.2$, the value close to the critical point estimated from the size dependence of the structure factor, the data are consistent with the linear convergence in $1 / \log L$ to the universal jump, as predicted in Ref. [29] as a characteristic size dependence for the KT transition. This may be taken as another piece of evidence suggesting the KT nature of the transition.

\section{THE MODEL WITH OTHER PARTICLE NUMBERS}

Let us briefly discuss the properties of the models with different particle numbers and a related issue about the charge gap.

When the number of particles $N$ is less than $|\mathcal{I}|$, we see that the space of the ground states is spanned by $\left|\Phi_{S}\right\rangle=$ $\left(\prod_{u \in S} \hat{b}_{u}^{\dagger}\right)\left|\Phi_{\text {vac }}\right\rangle$, where $S$ is an arbitrary subset of $\mathcal{I}$ such that $|S|=N$. The ground states show macroscopic degeneracy, which should be immediately lifted when a generic infinitesimal perturbation is added to the Hamiltonian.

The case with $N>|\mathcal{I}|$ is difficult, and we have almost no exact results. We can nevertheless construct a class of exact energy eigenstates, which may be regarded as examples of quantum many-body scars [30,31]. Assume that the lattice $(\mathcal{E}, \mathcal{B})$ is connected and bipartite. To be precise, we say that $(\mathcal{E}, \mathcal{B})$ is bipartite if there is a decomposition $\mathcal{E}=\mathcal{E}_{+} \cup \mathcal{E}_{-}$ such that two sites $x, y$ may be connected by a bond in $\mathcal{B}$ only when $x \in \mathcal{E}_{+}, y \in \mathcal{E}_{-}$or $x \in \mathcal{E}_{-}, y \in \mathcal{E}_{+}$. Let us define

$$
\hat{D}_{0}:=\sum_{x \in \mathcal{E}_{+}} \hat{a}_{x}-\sum_{x \in \mathcal{E}_{-}} \hat{a}_{x} .
$$

Then, for any subset $S \subset \mathcal{I}$ and $n=1,2 \ldots$, it is easily shown that the state

$$
\left|\Phi_{S, n}\right\rangle=\left(\hat{D}_{0}^{\dagger}\right)^{n}\left(\prod_{u \in S} \hat{b}_{u}^{\dagger}\right)\left|\Phi_{\text {vac }}\right\rangle,
$$

which has $|S|+n$ particles, is an energy eigenstate with eigenvalue $E_{n}=n t \zeta^{2}$. See Appendix E.
Let us denote by $E_{N}^{\mathrm{GS}}$ the ground-state energy of the model with $N$ particles and define the charge gap (or the jump in chemical potential) as

$$
\Delta_{N}=E_{N+1}^{\mathrm{GS}}+E_{N-1}^{\mathrm{GS}}-2 E_{N}^{\mathrm{GS}} .
$$

It is believed that the charge gap provides a simple criterion for conductivity in the sense that the ground state is insulating if $\Delta_{N}$ is positive and of order 1 [32].

Since we have $E_{N}^{\mathrm{GS}}=0$ for $N \leqslant|\mathcal{I}|$ in the present model, we see that $E_{|\mathcal{I}|+1}^{\mathrm{GS}}$ is nothing but the charge gap $\Delta_{|\mathcal{I}|}$, which is directly relevant to the property of the model with $|\mathcal{I}|$ particles. We conjecture that, for $\beta<\beta_{\mathrm{c}}$, the charge gap $\Delta_{|\mathcal{I}|}$ is strictly positive and our ground state describes a Mott insulator, while, for $\beta>\beta_{\mathrm{c}}$ where the ground state exhibits (quasi-) BEC, $\Delta_{|\mathcal{I}|}$ vanishes in the infinite volume limit according to the theorem in Ref. [33]. Therefore the ground state, although Mott-insulator-like, is not a genuine Mott insulator.

\section{DISCUSSION}

We proposed a class of exactly solvable models of interacting bosons with a flat band and argued that the Mottinsulator-like ground states may exhibit (quasi-)BEC. The conjecture is supported by strong numerical evidence that the two-dimensional model exhibits a KT transition. The properties of the three-dimensional models remain to be investigated.

We believe it important that an exactly solvable model that exhibits (or, that is conjectured to exhibit) nontrivial condensation phenomena has been discovered. It is also interesting to investigate the possibility of similar models of electrons, which should exhibit superconductivity.

It may be counterintuitive that our exact ground state (5), which consists of bosons almost localized at each $u \in \mathcal{I}$, exhibits ODLRO. One should note, however, that the operator $\hat{b}_{u}^{\dagger}$ creates a coherent superposition of the three states in which a particle is at $x, y$, and $u$. The coherence "propagates" in the system thus generating off-diagonal correlation, which may be short ranged or long ranged [15]. At least mathematically, the situation is parallel to that for the long-range Néel order in the exact valence-bond ground states of the AKLT model in high dimensions $[20,24,34]$.

Indeed, this point is related to the fact that the states proposed in Ref. [15] only have short-ranged off-diagonal correlation while our ground state on a suitable two-dimensional lattice exhibits quasi-ODLRO. The basic difference is not of a qualitative but of a quantitative nature; namely, the commutator $\left[\hat{b}_{u}, \hat{b}_{v}^{\dagger}\right]$ of neighboring sites, which give the basic parameter $\beta$, and the coordination number $v+1$ can be larger in our models compared with that in Ref. [15].

It is worth recalling that a two-dimensional system of interacting bosons at zero temperature generically exhibits genuine ODLRO rather than quasi-ODLRO. We believe that the present model exhibits only quasi-ODLRO because of its peculiar ground-state structure (5) expressed only in terms of local bosonic operators $\hat{b}_{u}^{\dagger}$. This is consistent with the fact that the ground state of the present two-dimensional model is represented in terms of a classical statistical mechanical model (i.e., the loop-gas model) in two dimensions, while a ground state in a two-dimensional quantum system generally corresponds to a three-dimensional classical system. We 


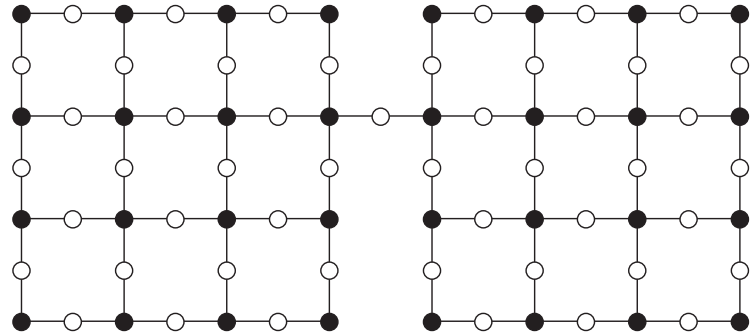

FIG. 8. The lattice obtained by connecting two lattices based on the square lattice by a single bond. The number of particles in each sublattice is almost constant in the corresponding ground state.

conjecture that the observed quasi-ODLRO will be immediately elevated into genuine ODLRO when the model is perturbed. See, e.g., Ref. [35] for a discussion of similar behavior in the ground-state correlation function of the onedimensional AKLT model.

We have repeatedly stressed that our exact ground state (5) has a Mott-insulator-like character since it is generated by local operators $\hat{b}_{u}^{\dagger}$. Its peculiar property is most clearly seen if one considers a special lattice $(\mathcal{E}, \mathcal{B})$ obtained by connecting two arbitrary standard lattices by a single bond. See Fig. 8. In the ground state of the model defined on the corresponding decorated lattice $\Lambda$, the number of particles in each of the two sublattices is almost constant. (To be precise, it fluctuates only by 1.) Note that this is also true when we properly choose the lattice so that the ground state exhibits (quasi-)ODLRO. Such a ground state with (quasi-)ODLRO and vanishing particlenumber fluctuation is quite exotic since (quasi-)ODLRO is usually accompanied by large density fluctuation. We should not, however, jump to the conclusion that a novel exotic phase of matter has been discovered. It is possible that the zero fluctuation is a singular property of the exactly solvable model and normal large fluctuation is recovered once the model is perturbed. For the moment we believe that this (less exciting) scenario is plausible. We nevertheless stress that the discovery of models in which anomalously small particle-number fluctuation and (quasi-)ODLRO may coexist indicates that strongly interacting systems of bosons may exhibit unexpectedly rich behavior.

\section{ACKNOWLEDGMENTS}

It is a pleasure to thank David Huse, Kensuke Tamura, and Itamar Kimchi for valuable comments. H.K. was supported by JSPS Grant-in-Aid for Scientific Research on Innovative Areas No. JP20H04630, JSPS Grant-in-Aid for Scientific Research No. JP18K03445, and the Inamori Foundation. N.K. was supported by JSPS Grant-in-Aid for Scientific Research No. JP19H01809. The numerical calculation is done on System B (ohtaka) of ISSP Supercomputer Center, The University of Tokyo.

\section{APPENDIX A: SINGLE-PARTICLE ENERGY EIGENVALUES}

Let us examine the band structure determined by the hopping Hamiltonian (2). We first recall that the states $\hat{b}_{u}^{\dagger}\left|\Phi_{\mathrm{vac}}\right\rangle$ with $u \in \mathcal{I}$ and $\hat{d}_{x}^{\dagger}\left|\Phi_{\text {vac }}\right\rangle$ with $x \in \mathcal{E}$ form a basis of the single- particle Hilbert space. Since the state $\hat{b}_{u}^{\dagger}\left|\Phi_{\text {vac }}\right\rangle$ has zero energy, we focus on states spanned by $\hat{d}_{x}^{\dagger}\left|\Phi_{\text {vac }}\right\rangle$.

Here, for simplicity, we assume that any pair $x, y \in \mathcal{E}$ is either connected by $p$ distinct bonds in $\mathcal{B}$ or not connected at all. We write $x \sim y$ when $x$ and $y$ are connected by $p$ bonds and denote by $\mathcal{E}(x)$ the set of $y \in \mathcal{E}$ such that $x \sim y$. Recalling the definition

$$
\hat{d}_{x}=\zeta \hat{a}_{x}+\sum_{u \in \mathcal{N}(x)} \hat{a}_{u}
$$

we see that

$$
\left[\hat{d}_{x}, \hat{d}_{y}^{\dagger}\right]= \begin{cases}\zeta^{2}+p z_{x} & x=y \\ p & x \sim y \\ 0 & \text { otherwise }\end{cases}
$$

where $z_{x}=|\mathcal{E}(x)|$ is the coordination number (i.e., the number of neighboring sites) of the original lattice $(\mathcal{E}, \mathcal{B})$. Note that $|\mathcal{N}(x)|=p z_{x}$. The commutation relation, along with the definition (2) of $\hat{H}_{\text {hop }}$, implies

$$
\left[\hat{H}_{\mathrm{hop}}, \hat{d}_{x}^{\dagger}\right]=\left(\zeta^{2}+p z_{x}\right) t \hat{d}_{x}^{\dagger}+p t \sum_{y \in \mathcal{E}(x)} \hat{d}_{y}^{\dagger} .
$$

Consider an arbitrary state spanned by $\hat{d}$ operators

$$
|\varphi\rangle=\sum_{x \in \mathcal{E}} \varphi_{x} \hat{d}_{x}^{\dagger}\left|\Phi_{\mathrm{vac}}\right\rangle
$$

where $\varphi_{x} \in \mathbb{C}$. By using the commutation relation (A3) and $\hat{H}_{\text {hop }}\left|\Phi_{\text {vac }}\right\rangle=0$, we see that

$$
\begin{aligned}
\hat{H}_{\mathrm{hop}}|\varphi\rangle & =\sum_{x \in \mathcal{E}} \varphi_{x}\left\{\left(\zeta^{2}+p z_{x}\right) t \hat{d}_{x}^{\dagger}+p t \sum_{y \in \mathcal{E}(x)} \hat{d}_{y}^{\dagger}\right\}\left|\Phi_{\mathrm{vac}}\right\rangle \\
& =\sum_{x \in \mathcal{E}}\left\{\left(\zeta^{2}+p z_{x}\right) t \varphi_{x}+p t \sum_{y \in \mathcal{E}(x)} \varphi_{y}\right\} \hat{d}_{x}^{\dagger}\left|\Phi_{\mathrm{vac}}\right\rangle
\end{aligned}
$$

where we switched the roles of $x$ and $y$ to get the final expression. Thus the Schrödinger equation

$$
\hat{H}_{\text {hop }}|\varphi\rangle=\epsilon|\varphi\rangle
$$

reduces to

$$
\left(\zeta^{2}+p z_{x}\right) t \varphi_{x}+p t \sum_{y \in \mathcal{E}(x)} \varphi_{y}=\epsilon \varphi_{x} \quad \text { for any } x \in \mathcal{E},
$$

which is nothing but the standard tight-binding Schrödinger equation with hopping $p t$ and on-site potential $\left(\zeta^{2}+p z_{x}\right) t$.

Now we suppose that $(\mathcal{E}, \mathcal{B})$ is the $d$-dimensional $L \times$ $\cdots \times L$ hypercubic lattice with even $L$ and periodic boundary conditions, i.e.,

$$
\mathcal{E}=\left\{\left(x_{1}, \ldots, x_{d}\right) \mid x_{j}=1, \ldots, L\right\},
$$

and $x \sim y$ when $|x-y|=1$. The coordination number is $z_{x}=$ $2 d$ for all $x$. Let us take the standard plane wave $\varphi_{x}^{(k)}=e^{i k \cdot x}$ with $k \cdot x=\sum_{j=1}^{d} k_{j} x_{j}$ and $k \in \mathcal{K}$, where the set of wavenumber vectors is

$$
\mathcal{K}=\left\{\left(k_{1}, \ldots, k_{d}\right) \mid k_{j}=\frac{2 \pi}{L} n_{j}, n_{j}=-L / 2+1, \ldots, L / 2\right\} .
$$


Substituting $\varphi_{x}^{(k)}$ into (A7), one readily confirms that this is an energy eigenstate with eigenvalue

$$
\epsilon(k)=\zeta^{2} t+2 p t \sum_{j=1}^{d}\left(\cos k_{j}+1\right) .
$$

See Fig. 2 for the case with $d=2$.

\section{APPENDIX B: LOOP-GAS REPRESENTATIONS}

Let us describe in some detail the derivations of the loopgas representations (8) and (9). From $\hat{b}_{u}\left|\Phi_{\text {vac }}\right\rangle=0$, we have the standard relation

$$
\begin{aligned}
\left\langle\Phi_{\mathrm{GS}} \mid \Phi_{\mathrm{GS}}\right\rangle & =\left\langle\Phi_{\mathrm{vac}}\left|\left(\prod_{u \in \mathcal{I}} \hat{b}_{u}\right)\left(\prod_{u \in \mathcal{I}} \hat{b}_{u}^{\dagger}\right)\right| \Phi_{\mathrm{vac}}\right\rangle \\
& =\sum_{\pi} \prod_{u \in \mathcal{I}}\left[\hat{b}_{u}, \hat{b}_{\pi(u)}^{\dagger}\right],
\end{aligned}
$$

where $\pi$ is summed over all permutations of the elements of $\mathcal{I}$.

We first focus on the simpler class of models where no sites $u, v \in \mathcal{I}$ satisfy $u \approx v$.

To see that (B1) leads to the claimed representation (8), it is best to first examine a simple example. Suppose that $\mathcal{I}=$ $\left\{u_{1}, u_{2}, u_{3}\right\}$, and it holds that $u_{1} \sim u_{2}, u_{2} \sim u_{3}$, and $u_{3} \sim u_{1}$. Then we see explicitly from (B1) that

$$
\begin{aligned}
\left\langle\Phi_{\mathrm{GS}} \mid \Phi_{\mathrm{GS}}\right\rangle= & 1+\left[\hat{b}_{1}, \hat{b}_{2}^{\dagger}\right]\left[\hat{b}_{2}, \hat{b}_{1}^{\dagger}\right]+\left[\hat{b}_{2}, \hat{b}_{3}^{\dagger}\right]\left[\hat{b}_{3}, \hat{b}_{2}^{\dagger}\right] \\
& +\left[\hat{b}_{3}, \hat{b}_{1}^{\dagger}\right]\left[\hat{b}_{1}, \hat{b}_{3}^{\dagger}\right]+\left[\hat{b}_{1}, \hat{b}_{2}^{\dagger}\right]\left[\hat{b}_{2}, \hat{b}_{3}^{\dagger}\right]\left[\hat{b}_{3}, \hat{b}_{1}^{\dagger}\right] \\
& +\left[\hat{b}_{1}, \hat{b}_{3}^{\dagger}\right]\left[\hat{b}_{3}, \hat{b}_{2}^{\dagger}\right]\left[\hat{b}_{2}, \hat{b}_{1}^{\dagger}\right] \\
= & 1+3 \beta^{2}+2 \beta^{3},
\end{aligned}
$$

where we abbreviated $\hat{b}_{u_{j}}$ as $\hat{b}_{j}$.

To treat general cases, fix $\pi$ and let $\mathcal{I}^{\prime}=\{u \in \mathcal{I} \mid \pi(u) \neq$ $u\}$. It is well known (and easily proved) that $\mathcal{I}^{\prime}$ is decomposed into a disjoint union as $\mathcal{I}^{\prime}=\bigcup_{k=1}^{n} c_{k}$, where each $c_{k}$ is a cycle. A cycle $c$ is a set of more than one site that can be rearranged into an ordered sequence $\left(u_{1}, \ldots, u_{m}\right)$ such that $\pi\left(u_{j}\right)=u_{j+1}$ for $j=1, \ldots, m$, where we wrote $u_{m+1}=u_{1}$. Defining the weight for the cycle $c$ as $W(c)=\prod_{j=1}^{m}\left[\hat{b}_{u_{j}}, \hat{b}_{u_{j+1}}^{\dagger}\right]$, we see that the summand on the right-hand side of (B1) factorizes as $\prod_{u \in \mathcal{I}}\left[\hat{b}_{u}, \hat{b}_{\pi(u)}^{\dagger}\right]=\prod_{k=1}^{n} W\left(c_{k}\right)$. By recalling (6), we get the desired representation (8).

To derive (9), we take arbitrary $v \neq u$ and evaluate

$$
\left\langle\Phi_{\mathrm{GS}}\left|\hat{a}_{v}^{\dagger} \hat{a}_{u}\right| \Phi_{\mathrm{GS}}\right\rangle=\zeta^{2}\left\langle\Phi_{\mathrm{vac}}\left|\left(\prod_{w \neq v} \hat{b}_{w}\right)\left(\prod_{w \neq u} \hat{b}_{w}^{\dagger}\right)\right| \Phi_{\mathrm{vac}}\right\rangle .
$$

Since there is $\hat{b}_{u}$ but no $\hat{b}_{u}^{\dagger}$, we must have $\left[\hat{b}_{u}, \hat{b}_{u_{1}}^{\dagger}\right]$ with $u \sim u_{1}$ to have a nonzero contribution. This implies that we also need $\left[\hat{b}_{u_{1}}, \hat{b}_{u_{2}}^{\dagger}\right]$ with $u_{1} \sim u_{2}$, and so on. This is terminated only when we have $\left[\hat{b}_{u_{m-1}}, \hat{b}_{v}^{\dagger}\right]$ with $u_{m-1} \sim v$. We have obtained the contribution from the random walk. The contribution from loops can be derived as in the above.

We now turn to a general class of models where we need to take into account the factor 2 that appears in the commutation relation $\left[\hat{b}_{u}, \hat{b}_{v}^{\dagger}\right]=2 \beta$ for $u, v \in \mathcal{I}$ such that $u \approx v$. This can
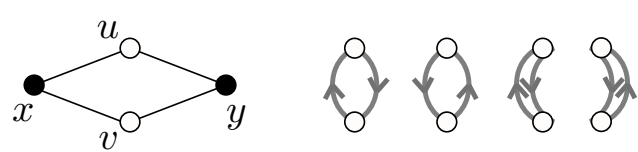

FIG. 9. The graphs corresponding to the normalization factor $\left\langle\Phi_{\mathrm{GS}} \mid \Phi_{\mathrm{GS}}\right\rangle$ in the simplest model with only two $u, v \in \mathcal{I}$ such that $u \approx v$.

be of course done by redefining the weights for loops and walks, but there is a more elegant way of incorporating the factor. We use the same definitions for the weights but regard in general that sites $u$ and $v$ with $u \approx v$ are connected by two distinct paths.

To see that this works, it (almost) suffices to consider the simplest model constructed from the lattice $\mathcal{E}=\{x, y\}$ with only two sites where $x$ and $y$ are connected by two distinct bonds. We then let $u$ and $v$ be the sites at the center of these bonds. We thus have $u \approx v$. In this case we see from (B1) that

$$
\left\langle\Phi_{\mathrm{GS}} \mid \Phi_{\mathrm{GS}}\right\rangle=1+\left[\hat{b}_{u}, \hat{b}_{v}^{\dagger}\right]\left[\hat{b}_{v}, \hat{b}_{u}^{\dagger}\right]=1+4 \beta^{2} .
$$

The factor 4 is reproduced as in Fig. 9.

The configurations of loops in these general models can be much more complicated than Fig. 3 in the main text. In the model with $d=2$ and $p=2$ depicted in Fig. 1(b), for example, the loops are defined on a lattice similar to Fig. 3, but each white site should be replaced by a pair of white sites connected by two distinct paths, and a path connecting two white sites should be replaced by four paths connecting two pairs of white sites.

\section{APPENDIX C: LARGE-d APPROXIMATION}

In the large- $d$ approximation, we can neglect the possibility that a random walk accidentally intersects its trajectory. This means that the number of length $n$ walks is given by $v^{n}$. We used this estimate in (16). Note that we here do not make a distinction between $v$ and $v+1$.

Let us see why we can neglect the contribution from loops. Let $\Omega(n)$ be the number of loops that contain a given site in $\mathcal{I}$. Since there are at most $(v+1)$ choices for the first step, at most $n$ choices for each of the following $n-2$ steps, and no choices in the final step, we have

$$
\Omega(n) \leqslant(v+1) v^{n-2} .
$$

Thus the total contribution from all the loops containing a site is bounded from above by

$$
\sum_{n=2}^{\infty} \Omega(n) \beta^{n} \lesssim \sum_{n=2}^{\infty} v^{n-1} \beta^{n}=\frac{1}{v} \sum_{n=2}^{\infty} \tilde{\beta}^{n} .
$$

This vanishes as $v \uparrow \infty$ provided that the sum converges. [To be precise, to fix a site and sum over all the loops containing it is not a proper way of evaluating the summations in (10). However, it gives a correct order estimate in terms of $v$.] 


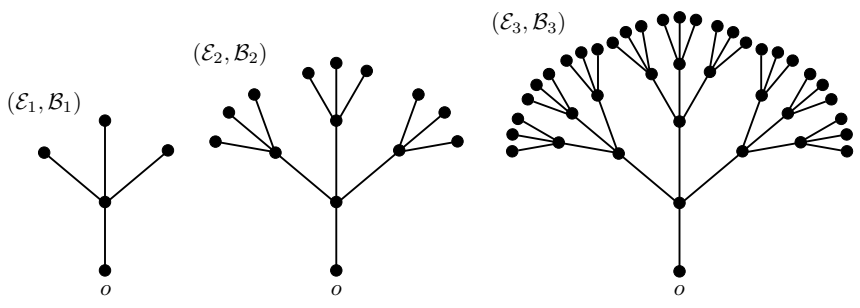

FIG. 10. The first three generations of the tree with branching number 3. The site at the root is denoted as $o$.

\section{APPENDIX D: BOSE-EINSTEIN CONDENSATION IN THE MODEL ON A TREE}

We shall study the model defined on a tree and show that the ground state exhibits Bose-Einstein condensation for sufficiently small $\zeta$.

Let $\left(\mathcal{E}_{n}, \mathcal{B}_{n}\right)$ be the regular $n$-generation tree with branching number 3 , as depicted in Fig. 10. As in the main text, we define the corresponding set of sites $\mathcal{I}_{n}$ and consider the model of interacting bosons on $\mathcal{E}_{n} \cup \mathcal{I}_{n}$. Our goal is to show that the ground state exhibits spontaneous symmetry breaking associated with BEC. We note that the model on the tree with branching number 2 does not exhibit a phase transition.

A standard method to test for the existence of spontaneous symmetry breaking is to impose boundary conditions that explicitly favor certain order and see whether the effect of the boundary conditions survives in the infinite-volume limit. In the case of ferromagnetic spin systems, this is done by enforcing spins at the boundary to point in a certain fixed direction. In the case of BEC, the corresponding procedure is to replace $\hat{b}_{u}^{\dagger}$ at the boundary by $\alpha+\gamma \hat{b}_{u}^{\dagger}$ with some nonzero $\alpha, \gamma \in \mathbb{C}$ and then to examine the expectation value of the annihilation operator deep inside the tree. In the ferromagnetic Ising model, for example, it is known that the same procedure exactly recovers the result of the Bethe approximation [23]. See also Ref. [24] for a treatment of a quantum spin state.

To be precise, let $\partial \mathcal{I}_{n}$ be the set of sites at the boundary in $\mathcal{I}_{n}$. See Fig. 11. We then consider the ground state with the plus boundary condition defined as

$$
\left|\Phi_{\mathrm{GS}}^{+}\right\rangle=\left(\prod_{u \in \mathcal{I}_{n} \backslash \partial \mathcal{I}_{n}} \hat{b}_{u}^{\dagger}\right)\left(\prod_{u \in \partial \mathcal{I}_{n}}\left(1+\hat{b}_{u}^{\dagger}\right)\right)\left|\Phi_{\mathrm{vac}}\right\rangle
$$

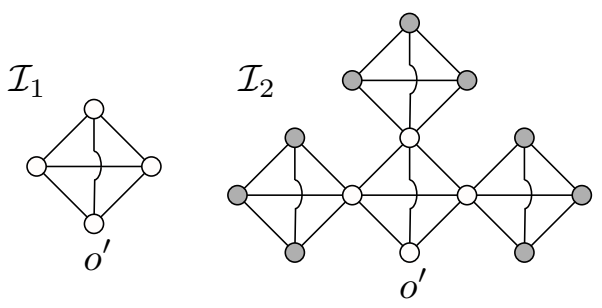

FIG. 11. The lattices $\mathcal{I}_{1}$ and $\mathcal{I}_{2}$. The bonds denote the connection $u \sim u^{\prime}$. The gray circles are the sites in the boundary $\partial \mathcal{I}_{n}$, and the root of the lattice is denoted as $o^{\prime}$.
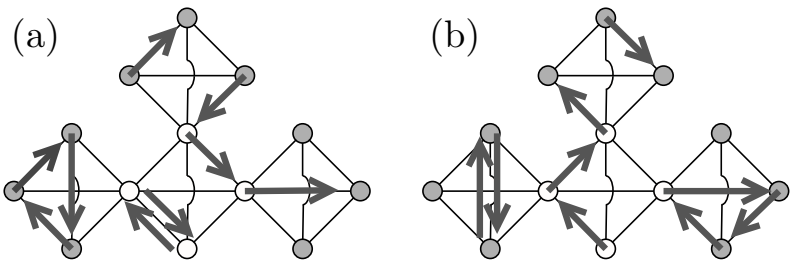

FIG. 12. Allowed configurations of loops and walks for the representations of (a) $\left\langle\Phi_{\mathrm{GS}}^{+} \mid \Phi_{\mathrm{GS}}^{+}\right\rangle$and (b) $\left\langle\Phi_{\mathrm{GS}}^{+}\left|\hat{a}_{o}\right| \Phi_{\mathrm{GS}}^{+}\right\rangle$.

Note that we have chosen $\alpha=\gamma=1$ for simplicity. We are interested in the expectation value

$$
\left\langle\hat{a}_{o}\right\rangle_{\mathrm{GS}}^{+}=\frac{\left\langle\Phi_{\mathrm{GS}}^{+}\left|\hat{a}_{o}\right| \Phi_{\mathrm{GS}}^{+}\right\rangle}{\left\langle\Phi_{\mathrm{GS}}^{+} \mid \Phi_{\mathrm{GS}}^{+}\right\rangle},
$$

especially in its limiting value as $n \uparrow \infty$, where $o$ denotes the site at the root of the tree $\left(\mathcal{E}_{n}, \mathcal{B}_{n}\right)$.

As in the main text, we develop loop-gas representations for $\left\langle\Phi_{\mathrm{GS}}^{+} \mid \Phi_{\mathrm{GS}}^{+}\right\rangle$and $\left\langle\Phi_{\mathrm{GS}}^{+}\left|\hat{a}_{o}\right| \Phi_{\mathrm{GS}}^{+}\right\rangle$. Reflecting the special geometry of $\mathcal{I}_{n}$, the representations contain loops of length 2 , 3 , or 4 , but not larger. Apart from these loops the representations contain random walks that start from a site in $\partial \mathcal{I}_{n}$ and end at another site in $\partial \mathcal{I}_{n}$. Note that the symmetry-breaking boundary terms play the roles of sources and sinks of the walks. Of course, the loops and the walks should satisfy the site-avoiding conditions. See Fig. 12(a). These are all contributions to the representation for $\left\langle\Phi_{\mathrm{GS}}^{+} \mid \Phi_{\mathrm{GS}}^{+}\right\rangle$, and we have

$$
\left\langle\Phi_{\mathrm{GS}}^{+} \mid \Phi_{\mathrm{GS}}^{+}\right\rangle=\sum_{m=0}^{\infty} \sum_{\ell_{1}, \ldots, \ell_{m}} \sum_{k=0}^{\infty} \sum_{\omega_{1}, \ldots, \omega_{k}: \partial \mathcal{I} \rightarrow \partial \mathcal{I}} \beta^{\sum\left|\ell_{j}\right|+\sum\left|\omega_{j}\right|} .
$$

The representation of $\left\langle\Phi_{\mathrm{GS}}^{+}\left|\hat{a}_{o}\right| \Phi_{\mathrm{GS}}^{+}\right\rangle$must contain a random walk that starts from site $o^{\prime}$, the root of $\mathcal{I}_{n}$, and ends at a site in $\partial \mathcal{I}_{n}$. See Fig. 12(b). Thus the representation is given by

$$
\begin{aligned}
\left\langle\Phi_{\mathrm{GS}}^{+}\left|\hat{a}_{o}\right| \Phi_{\mathrm{GS}}^{+}\right\rangle= & \sqrt{\beta} \sum_{\omega_{0}: o^{\prime} \rightarrow \partial \mathcal{I}} \sum_{m=0}^{\infty} \sum_{\ell_{1}, \ldots, \ell_{m}} \\
& \times \sum_{k=0}^{\infty} \sum_{\omega_{1}, \ldots, \omega_{k}: \partial \mathcal{I} \rightarrow \partial \mathcal{I}} \beta^{\sum\left|\ell_{j}\right|+\sum\left|\omega_{j}\right|} .
\end{aligned}
$$

Following the standard procedure for models on a tree $[23,24]$, we shall evaluate these sums by using exact recursion relations. We define four sums $X_{n}, Y_{n}, Z_{n}^{+}$, and $Z_{n}^{-}$of loops and walks as in (D3) and (D4) with different conditions on site $o^{\prime}$ at the root of $\mathcal{I}_{n}$. In $X_{n}$ we sum over all configurations in which there is no loop or walk touching $o^{\prime}$. In $Y_{n}$ we sum over all configurations in which there are two segments (which are part of a loop or a walk) touching $o^{\prime}$. In $Z_{n}^{+}\left(Z_{n}^{-}\right)$we sum over all configurations where there is exactly one segment (which is a part of a walk) coming into (going out of) $o^{\prime}$. Note that we have $X_{0}=1, Y_{0}=0$, and $Z_{0}^{ \pm}=1$. We see that $\left\langle\Phi_{\mathrm{GS}}^{+} \mid \Phi_{\mathrm{GS}}^{+}\right\rangle=X_{n}+Y_{n},\left\langle\Phi_{\mathrm{GS}}^{+}\left|\hat{a}_{o}\right| \Phi_{\mathrm{GS}}^{+}\right\rangle=\sqrt{\beta} Z_{n}^{-}$, and hence

$$
\left\langle\hat{a}_{o}\right\rangle_{\mathrm{GS}}^{+}=\sqrt{\beta} \frac{Z_{n}^{-}}{X_{n}+Y_{n}}=\sqrt{\beta} \frac{z_{n}}{1+y_{n}},
$$




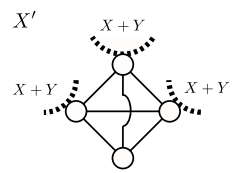

$(X+Y)^{3}$

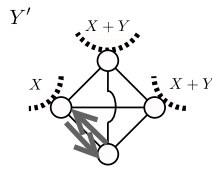

$3 \beta^{2} X(X+Y)^{2}$

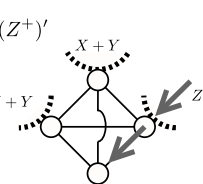

$3 \beta(X+Y)^{2} Z$

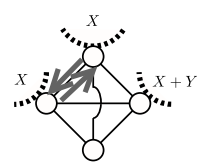

$3 \beta^{2} X^{2}(X+Y)$

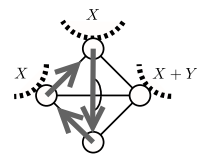

$6 \beta^{3} X^{2}(X+Y)$

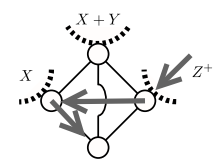

$6 \beta^{2} X(X+Y) Z^{+}$

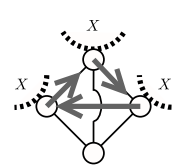

$2 \beta^{3} X^{3}$

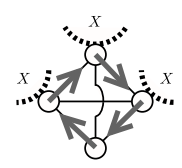

$6 \beta^{4} X^{3}$

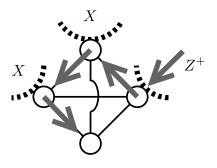

$6 \beta^{3} X^{2} Z^{+}$

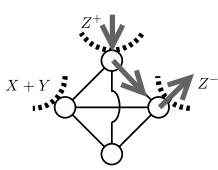

$6 \beta(X+Y) Z^{+} Z^{-}$

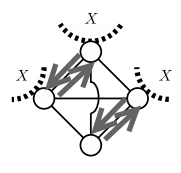

$3 \beta^{4} X^{3}$

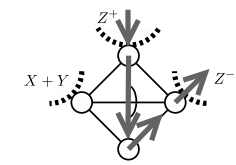

$6 \beta^{2}(X+Y) Z^{+} Z^{-}$
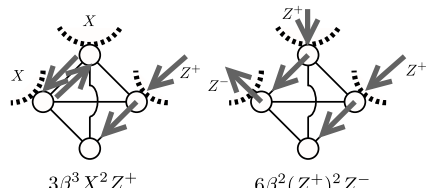

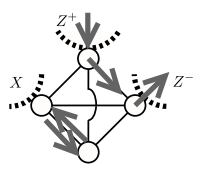

$6 \beta^{3} X Z^{+} Z^{-}$

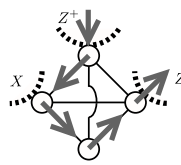

$6 \beta^{3} X Z^{+} Z^{-}$

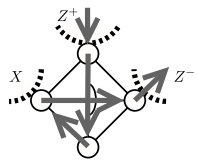

$6 \beta^{3} X Z^{+} Z^{-}$

FIG. 13. The diagrammatic derivation of the recursion relations (D7), (D8), and (D9). $\mathcal{I}_{n+1}$ is decomposed into the square containing the root $o^{\prime}$ and three copies of $\mathcal{I}_{n}$. By specifying a configuration (of loops and walks) on the square, possible types of configurations on each branch are determined separately. By counting the number of similar configurations, we get the recursion relations.

where we defined

$$
y_{n}=\frac{Y_{n}}{X_{n}}, \quad z_{n}=\frac{Z_{n}^{-}}{X_{n}} .
$$

Now it is straightforward (although tedious) to see that $X_{n}, Y_{n}$, $Z_{n}^{+}$, and $Z_{n}^{-}$satisfy the exact recursion relations

$$
\begin{gathered}
X^{\prime}=(X+Y)^{3}+3 \beta^{2} X^{2}(X+Y)+2 \beta^{3} X^{3} \\
+6 \beta(X+Y) Z^{+} Z^{-}+6 \beta^{2} X Z^{+} Z^{-}, \\
Y^{\prime}=3 \beta^{2} X(X+Y)^{2}+6 \beta^{3} X^{2}(X+Y)+6 \beta^{4} X^{3}+3 \beta^{4} X^{3} \\
+6 \beta^{2}(X+Y) Z^{+} Z^{-}+18 \beta^{3} X Z^{+} Z^{-}, \\
\left(Z^{+}\right)^{\prime}=3 \beta(X+Y)^{2} Z^{+}+6 \beta^{2} X(X+Y) Z^{+} \\
+9 \beta^{3} X^{2} Z^{+}+6 \beta^{2}\left(Z^{+}\right)^{2} Z^{-}, \\
\left(Z^{-}\right)^{\prime}= \\
3 \beta(X+Y)^{2} Z^{-}+6 \beta^{2} X(X+Y) Z^{-} \\
+9 \beta^{3} X^{2} Z^{-}+6 \beta^{2} Z^{+}\left(Z^{-}\right)^{2},
\end{gathered}
$$

where we wrote $X_{n-1}, Y_{n-1}$, and $Z_{n-1}^{ \pm}$as $X, Y$, and $Z^{ \pm}$, and $X_{n}$, $Y_{n}$, and $Z_{n}^{ \pm}$as $X^{\prime}, Y^{\prime}$, and $\left(Z^{ \pm}\right)^{\prime}$. See Fig. 13. Since (D9) and (D10) are symmetric under the exchange of $Z^{+}$and $Z^{-}$and we have $Z_{0}^{+}=Z_{0}^{-}$, we see that $Z_{n}^{+}=Z_{n}^{-}$for all $n$.

Then the relations (D7), (D8), (D9), and (D10) lead to the following recursion relations for $y_{n}$ and $z_{n}=Z_{n}^{ \pm} / X_{n}$ :

$$
\begin{aligned}
y^{\prime} & =\frac{3 \beta^{2}(1+y)^{2}+6 \beta^{3}(1+y)+9 \beta^{4}+6 \beta^{2}(1+y) z^{2}+18 \beta^{3} z^{2}}{(1+y)^{3}+3 \beta^{2}(1+y)+2 \beta^{3}+6 \beta(1+y) z^{2}+6 \beta^{2} z^{2}}, \\
z^{\prime} & =\frac{3 \beta(1+y)^{2} z+6 \beta^{2}(1+y) z+9 \beta^{3} z+6 \beta^{2} z^{3}}{(1+y)^{3}+3 \beta^{2}(1+y)+2 \beta^{3}+6 \beta(1+y) z^{2}+6 \beta^{2} z^{2}},
\end{aligned}
$$

where we wrote $y_{n-1}$ and $z_{n-1}$ as $y$ and $z$, and $y_{n}$ and $z_{n}$ as $y^{\prime}$ and $z^{\prime}$. Our task is to start from the initial values $\left(y_{0}, z_{0}\right)=$ $(0,1)$, repeatedly apply the recursion relations (D11) and (D12), and find the behavior of $\left(y_{n}, z_{n}\right)$ in the limit $n \uparrow \infty$. We get a reliable conclusion from a simple numerical calculation.
We see that there is a critical value of $\beta$, which is estimated to be $\beta_{\mathrm{c}} \simeq 0.319$. For $\beta \in\left(0, \beta_{\mathrm{c}}\right)$, we see that $y_{n} \rightarrow y^{*}(\beta)>$ 0 and $z_{n} \rightarrow 0$. This means that the order parameter $\left\langle\hat{a}_{o}\right\rangle_{\mathrm{GS}}^{+}$ tends to zero as $n \uparrow \infty$, indicating that there is no BEC. For $\beta \in\left(\beta_{\mathrm{c}}, 1 / 2\right)$, we see that $y_{n} \rightarrow y^{*}(\beta)>0$ and $z_{n} \rightarrow z^{*}(\beta)>$ 0 . Thus the order parameter $\left\langle\hat{a}_{o}\right\rangle_{\mathrm{GS}}^{+}$converges to a nonzero value as $n \uparrow \infty$. This means that the ground state exhibits spontaneous symmetry breaking of the U(1) symmetry, which corresponds to BEC.

\section{APPENDIX E: EXACT ENERGY EIGENSTATES}

We construct a series of exact energy eigenstates for any particle number, including $N=|\mathcal{I}|$. These energy eigenstates are interesting by themselves since they provide examples of quantum many-body scars [30,31].

Here, we assume that the lattice $(\mathcal{E}, \mathcal{B})$ is connected and bipartite. By saying that $(\mathcal{E}, \mathcal{B})$ is bipartite we mean that there is a decomposition $\mathcal{E}=\mathcal{E}_{+} \cup \mathcal{E}_{-}$such that two sites $x$, y may be connected by a bond in $\mathcal{B}$ only when $x \in \mathcal{E}_{+}, y \in \mathcal{E}_{-}$or $x \in$ $\mathcal{E}_{-}, y \in \mathcal{E}_{+}$. For simplicity we shall assume that a pair of sites $x, y \in \mathcal{E}$ is either connected by $p$ bonds in $\mathcal{B}$ or not connected at all. However, the following result regarding exact energy eigenstates is valid without this restriction.

As in Sec. V, we define

$$
\hat{D}_{0}:=\sum_{x \in \mathcal{E}_{+}} \hat{a}_{x}-\sum_{x \in \mathcal{E}_{-}} \hat{a}_{x} .
$$

Then we shall prove, for any subset $S \subset \mathcal{I}$ and $n=1,2, \ldots$, that the state

$$
\left|\Phi_{S, n}\right\rangle=\left(\hat{D}_{0}^{\dagger}\right)^{n}\left(\prod_{u \in S} \hat{b}_{u}^{\dagger}\right)\left|\Phi_{\mathrm{vac}}\right\rangle
$$

satisfies

$$
\hat{H}\left|\Phi_{S, n}\right\rangle=n t \zeta^{2}\left|\Phi_{S, n}\right\rangle .
$$

Thus $\left|\Phi_{S, n}\right\rangle$ is an exact energy eigenstate with particle number $N=|S|+n$ and energy $E_{S, n}=n t \zeta^{2}$. 
Note that we can express $\hat{D}_{0}$ in terms of the $\hat{d}$ operators as

$$
\hat{D}_{0}=\frac{1}{\zeta}\left(\sum_{x \in \mathcal{E}_{+}} \hat{d}_{x}-\sum_{x \in \mathcal{E}_{-}} \hat{d}_{x}\right) .
$$

Then, by using (A3), we find

$$
\begin{aligned}
{\left[\hat{H}_{\mathrm{hop}}, \hat{D}_{0}^{\dagger}\right] } & =\frac{1}{\zeta}\left(\sum_{x \in \mathcal{E}_{+}}\left\{t\left(\zeta^{2}+p z_{x}\right) \hat{d}_{x}^{\dagger}+p t \sum_{y \in \mathcal{E}(x)} \hat{d}_{y}^{\dagger}\right\}-\sum_{x \in \mathcal{E}_{-}}\left\{t\left(\zeta^{2}+p z_{x}\right) \hat{d}_{x}^{\dagger}+p t \sum_{y \in \mathcal{E}(x)} \hat{d}_{y}^{\dagger}\right\}\right) \\
& =\frac{1}{\zeta}\left(\sum_{x \in \mathcal{E}_{+}} t \zeta^{2} \hat{d}_{x}^{\dagger}-\sum_{x \in \mathcal{E}_{-}} t \zeta^{2} \hat{d}_{x}^{\dagger}\right) \\
& =t \zeta^{2} \hat{D}_{0}^{\dagger} .
\end{aligned}
$$

We note in passing that $t \zeta^{2}$ is the minimum energy among single-particle energy eigenstates that are orthogonal to the flat band (i.e., the states generated by the $\hat{b}^{\dagger}$ operators) and that the state generated by $\hat{D}_{0}^{\dagger}$ is the unique energy eigenstate with energy $t \zeta^{2}$. This fact is obvious from the dispersion relation (A10) for the models on the hypercubic lattice but can be proved in general.
Note that by construction the operator $\hat{D}_{0}^{\dagger}$ does not contain $\hat{a}_{u}^{\dagger}$ for any $u \in \mathcal{I}$. This immediately implies that

$$
\left[\hat{H}_{\text {int }}, \hat{D}_{0}^{\dagger}\right]=0 .
$$

It is clear from (E5) and (E6) that $\hat{H}_{\mathrm{hop}}\left|\Phi_{S, n}\right\rangle=n t \zeta^{2}\left|\Phi_{S, n}\right\rangle$ and $\hat{H}_{\text {int }}\left|\Phi_{S, n}\right\rangle=0$. We thus find that $\left|\Phi_{S, n}\right\rangle$ is an eigenstate of $\hat{H}=\hat{H}_{\text {hop }}+\hat{H}_{\text {int }}$ with eigenvalue $E_{S, n}=n t \zeta^{2}$.
[1] E. H. Lieb, Two Theorems on the Hubbard Model, Phys. Rev. Lett. 62, 1201 (1989).

[2] A. Mielke, Ferromagnetism in the Hubbard model on line graphs and further considerations, J. Phys. A: Math. Gen. 24, 3311 (1991).

[3] A. Mielke, Exact ground states for the Hubbard model on the Kagome lattice, J. Phys. A: Math. Gen. 25, 4335 (1992).

[4] H. Tasaki, Ferromagnetism in the Hubbard Models with Degenerate Single-Electron Ground States, Phys. Rev. Lett. 69, 1608 (1992).

[5] A. Mielke and H. Tasaki, Ferromagnetism in the Hubbard model. Examples from models with degenerate single-electron ground states, Commun. Math. Phys. 158, 341 (1993).

[6] H. Tasaki, Physics and Mathematics of Quantum Many-Body Systems, Graduate Texts in Physics (Springer, New York, 2020).

[7] S. D. Huber and E. Altman, Bose condensation in flat bands, Phys. Rev. B 82, 184502 (2010).

[8] S. Takayoshi, H. Katsura, N. Watanabe, and H. Aoki, Phase diagram and pair Tomonaga-Luttinger liquid in a Bose-Hubbard model with flat bands, Phys. Rev. A 88, 063613 (2013).

[9] M. Tovmasyan, E. van Nieuwenburg, and S. Huber, Geometry induced pair condensation, Phys. Rev. B 88, 220510(R) (2013).

[10] A. Mielke, Pair formation of hard core bosons in flat band systems, J. Stat. Phys. 171, 679 (2018).

[11] J. Fronk and A. Mielke, Localised pair formation in bosonic flat-band Hubbard models, arXiv:2008.01756 [math-ph].

[12] Y. Cao, V. Fatemi, S. Fang, K. Watanabe, T. Taniguchi, E. Kaxiras, and P. Jarillo-Herrero, Unconventional superconductivity in magic-angle graphene superlattices, Nature (London) 556, 43 (2018).
[13] H. Tasaki, From Nagaoka's ferromagnetism to flat-band ferromagnetism and beyond: An introduction to ferromagnetism in the Hubbard model, Prog. Theor. Phys. 99, 489 (1998).

[14] H. Yang, H. Nakano, and H. Katsura, Symmetry-protected topological phases in spinful bosons with a flat band, Phys. Rev. Research 3, 023210 (2021).

[15] I. Kimchi, S. A. Parameswaran, A. M. Turner, F. Wang, and A. Vishwanath, Featureless and non-fractionalized Mott insulators on the honeycomb lattice at $1 / 2$ site filling, Proc. Natl. Acad. Sci. U. S. A. 110, 16378 (2013).

[16] G.-H. Chen and Y.-S. Wu, Quantum phase transition in a multicomponent Bose-Einstein condensate in optical lattices, Phys. Rev. A 67, 013606 (2003).

[17] E. Kim and M. H. W. Chan, Observation of superflow in solid helium, Science 305, 1941 (2004).

[18] T. Ohgoe, T. Suzuki, and N. Kawashima, Commensurate Supersolid of Three-Dimensional Lattice Bosons, Phys. Rev. Lett. 108, 185302 (2012).

[19] R. Suzuki and A. Koga, Supersolid states in a hard-core BoseHubbard model on a layered triangular lattice, J. Phys. Soc. Jpn. 83, 064003 (2014).

[20] T. Kennedy, E. H. Lieb, and H. Tasaki, A two-dimensional isotropic quantum antiferromagnet with unique disordered ground state, J. Stat. Phys. 53, 383 (1988).

[21] H.-Y. Lee, R. Kaneko, T. Okubo, and N. Kawashima, Gapless Kitaev Spin Liquid to Classical String Gas through Tensor Networks, Phys. Rev. Lett. 123, 087203 (2019).

[22] M. Aizenman, E. H. Lieb, R. Seiringer, J. P. Solovej, and J. Yngvason, Bose-Einstein quantum phase transition in an optical lattice model, Phys. Rev. A 70, 023612 (2004). 
[23] C. J. Thompson, Local properties of an Ising model on a Cayley tree, J. Stat. Phys. 27, 441 (1982).

[24] I. Affleck, T. Kennedy, E. H. Lieb, and H. Tasaki, Valence bond ground states in isotropic quantum antiferromagnets, Commun. Math. Phys. 115, 477 (1988).

[25] N. V. Prokof'ev, B. V. Svistunov, and I. S. Tupitsyn, "Worm" algorithm in quantum Monte Carlo simulations, Phys. Lett. A 238, 253 (1998).

[26] J. M. Kosterlitz, The critical properties of the two-dimensional xy model, J. Phys. C: Solid State Phys. 7, 1046 (1974).

[27] E. L. Pollock and D. M. Ceperley, Path-integral computation of superfluid densities, Phys. Rev. B 36, 8343 (1987).

[28] W. Janke, Logarithmic corrections in the two-dimensional XY model, Phys. Rev. B 55, 3580 (1997).

[29] H. Weber and P. Minnhagen, Monte Carlo determination of the critical temperature for the two-dimensional XY model, Phys. Rev. B 37, 5986(R) (1988).
[30] M. Serbyn, D. A. Abanin, and Z. Papić, Quantum many-body scars and weak breaking of ergodicity, Nat. Phys. 17, 675 (2021).

[31] Y. Kuno, T. Mizoguchi, and Y. Hatsugai, Flat band quantum scar, Phys. Rev. B 102, 241115(R) (2020).

[32] E. H. Lieb and F. Y. Wu, Absence of Mott Transition in an Exact Solution of the Short-Range, One-Band Model in One Dimension, Phys. Rev. Lett. 20, 1445 (1968); 21, 192(E) (1968).

[33] H. Tasaki and H. Watanabe, Off-diagonal long-range order implies vanishing charge gap, arXiv:2105.10692 [cond-mat.quantgas].

[34] D. P. Arovas, A. Auerbach, and F. D. M. Haldane, Extended Heisenberg Models of Antiferromagnetism: Analogies to the Fractional Quantum Hall Effect, Phys. Rev. Lett. 60, 531 (1988).

[35] K. Nomura, Onset of incommensurability in quantum spin chains, J. Phys. Soc. Jpn. 72, 476 (2003). 